International Mathematical Forum, Vol. 8, 2013, no. 7, 311 - 317

\title{
Second-Order Domain Decomposition Method for Three-Dimensional Hyperbolic Problems
}

\author{
Younbae Jun \\ Department of Applied Mathematics \\ Kumoh National Institute of Technology \\ Gumi, Gyeongbuk 730-701, Korea \\ yjun@kumoh.ac.kr
}

\begin{abstract}
In this paper, a non-overlapping second-order domain decomposition method for solving three-dimensional hyperbolic partial differential equation is proposed. Unconditional stability of the algorithm is analyzed. Numerical experiments show that the method is stable and very efficient.
\end{abstract}

Mathematics Subject Classification: 65M06, 65M55

Keywords: three-dimensional hyperbolic problem, domain decomposition, parallel algorithm, stability, efficiency

\section{Introduction}

Hyperbolic partial differential equation describes a number of interesting physical problems in diverse areas such as: fluid dynamics and aerodynamics, solid mechanics, astrophysics, the theory of elasticity, optics, electromagnetic waves, direct and inverse scattering, and the general theory of relativity. In this paper, we consider the three-dimensional linear second-order hyperbolic partial differential equation of the form

$$
\begin{aligned}
u_{t t}+a(x, y, z, t) u_{t}+b(x, y, z, t) u & =c(x, y, z, t) u_{x x}+d(x, y, z, t) u_{y y} \\
& +e(x, y, z, t) u_{z z}+f(x, y, z, t)
\end{aligned}
$$

defined in $\{(x, y, z, t) \mid 0 \leq x, y, z \leq 1,0 \leq t \leq T\}$, with initial conditions

$$
u(x, y, z, 0)=\phi(x, y, z), u_{t}(x, y, z, 0)=\psi(x, y, z) \text { in } \Omega
$$

This paper was supported by Research Fund, Kumoh National Institute of Technology. 
and boundary condition

$$
u(x, y, z, t)=g(x, y, z, t) \text { on the boundary } \partial \Omega, 0 \leq t \leq T,
$$

where $\partial \Omega$ is the boundary of $\Omega=\{(x, y, z) \mid 0 \leq x, y, z \leq 1\}$.

There are many implementations on hyperbolic problems such as: finite volume method with grid refinement technique [2], finite difference scheme for adaptive mesh refinement [5], two-time level alternating direction implicit (ADI) finite volume scheme [9], and many others. For three-dimensional hyperbolic problems, there are Galerkin alternating-direction method [6], unconditionally stable ADI method [7], operator splitting technique [8]. However, most of these methods emphasized mainly on the stability, not on the efficiency.

Domain decomposition (DD) is one of the techniques for solving partial differential equation. The method is very efficient especially when a parallel computer is used. In the method, the original spatial domain is decomposed into subdomains and the pde in each subdomain is solved in parallel manner. Jun $[3,4]$ has proposed unconditionally stable DD methods for linear second-order hyperbolic equations in one and two space dimensions. In general, implementation of DD method to solve high dimensional hyperbolic problem is somewhat complicated. So far, there is no literature dealing with three-dimensional hyperbolic problem using efficient domain decomposition technique.

In this paper, we focus on domain decomposition method to solve three dimensional hyperbolic problem. The paper is organized as follows. In the next section we introduce new algorithm and analyze its stability. In Section 3, numerical results on the performance of the method are reported with respect to accuracy and efficiency. Then we make concluding remarks in Section 4.

\section{Algorithm and Stability}

In this section, we describe new domain decomposition algorithm to solve the problem (1)-(3). A finite difference scheme is used to discretize the partial differential equation with the central difference formulas. We let $w_{i, j, k}^{n}$ be the approximated value to the exact value $u_{i, j, k}^{n}$ at the grid point $\left(x_{i}, y_{j}, z_{k}, t_{n}\right)$, where $\Delta x=\frac{1}{I}, \Delta y=\frac{1}{J}, \Delta z=\frac{1}{K}, \Delta t=\frac{T}{N}$, for some preset positive integers $I$, $J, K, N$ and $x_{i}=i \Delta x, y_{j}=j \Delta y, z_{k}=k \Delta z, t_{n}=n \Delta t$. We define the finite

difference operators at the grid point $\left(x_{i}, y_{j}, z_{k}, t_{n}\right)$ as $w_{t t}^{n}=\frac{w_{i, j, k}^{n+1}-2 w_{i, j, k}^{n}+w_{i, j, k}^{n-1}}{(\Delta t)^{2}}$, $w_{t}^{n}=\frac{w_{i, j, k}^{n+1}-w_{i, j, k}^{n-1}}{2 \Delta t}, w_{x x}^{n}=\frac{w_{i+1, j, k}^{n}-2 w_{i, j, k}^{n}+w_{i-1, j, k}^{n}}{(\Delta x)^{2}}, w_{y y}^{n}=\frac{w_{i, j+1, k}^{n}-2 w_{i, j, k}^{n}+w_{i, j-1, k}^{n}}{(\Delta y)^{2}}$, $w_{z z}^{n}=\frac{w_{i, j, k+1}^{n}-2 w_{i, j, k}^{n}+w_{i, j, k-1}^{n}}{(\Delta z)^{2}}$. And we denote $a\left(x_{i}, y_{j}, z_{k}, t_{n}\right), b\left(x_{i}, y_{j}, z_{k}, t_{n}\right)$, $c\left(x_{i}, y_{j}, z_{k}, t_{n}\right), d\left(x_{i}, y_{j}, z_{k}, t_{n}\right), e\left(x_{i}, y_{j}, z_{k}, t_{n}\right)$, and $f\left(x_{i}, y_{j}, z_{k}, t_{n}\right)$, by $a_{i j k}^{n}, b_{i j k}^{n}$, $c_{i j k}^{n}, d_{i j k}^{n}, e_{i j k}^{n}$, and $f_{i j k}^{n}$, respectively. 
It is well-known [1] that the classical fully explicit three-level finite difference scheme referred to as the fully explicit scheme (FES) is conditionally stable and the scheme can be written as follows:

$$
w_{t t}^{n}+a_{i j k}^{n} w_{t}^{n}+b_{i j k}^{n} w_{i j k}^{n}=c_{i j k}^{n} w_{x x}^{n}+d_{i j k}^{n} w_{y y}^{n}+e_{i j k}^{n} w_{z z}^{n}+f_{i j k}^{n}
$$

and the classical fully implicit three-level scheme referred to as the fully implicit scheme (FIS) is unconditionally stable, which can be written as

$$
\begin{aligned}
w_{t t}^{n}+a_{i j k}^{n} w_{t}^{n}+b_{i j k}^{n} w_{i j k}^{n} & =c_{i j k}^{n} \frac{1}{2}\left[w_{x x}^{n+1}+w_{x x}^{n-1}\right]+d_{i j k}^{n} \frac{1}{2}\left[w_{y y}^{n+1}+w_{y y}^{n-1}\right] \\
& +e_{i j k}^{n} \frac{1}{2}\left[w_{z z}^{n+1}+w_{z z}^{n-1}\right]+f_{i j k}^{n} .
\end{aligned}
$$

These FES and FIS are used to be compared with our new scheme, in this paper.

Now we describe new finite difference scheme to solve hyperbolic problem (1)-(3) using domain decomposition technique. Suppose, for the simplicity, we decompose the spatial domain into non-overlapping stripwise subdomains along the $x$-direction shown in Figure 1 . Then it is easy to see that the adjacent subdomains share an interface plane.

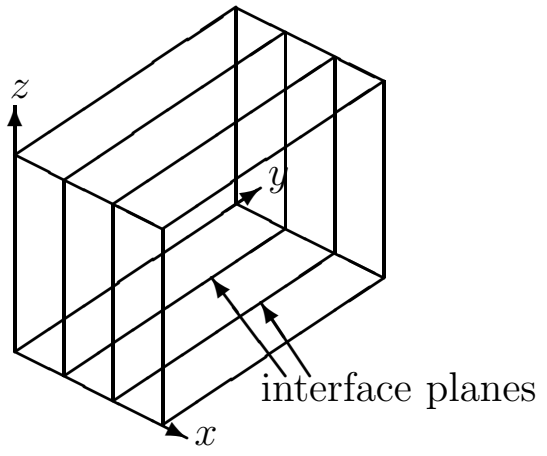

Figure 1. Stripwise Decomposition

In order to solve each divided subdomain problem independently, the values at the interface points need to be estimated in advance. Under uniformly spaced interface planes assumption, the distance between adjacent interface planes is $\frac{1}{P}$ if the domain is decomposed into $P$ subdomains. Let $H=\frac{1}{P}$. Then using the central difference scheme for $u(x, y, z, t)$, we obtain $u_{x x}(x, y, z, t)=$ $\frac{u(x+H, y, z, t)-2 u(x, y, z, t)+u(x-H, y, z, t)}{H^{2}}+O\left(H^{2}\right)$ at the interface plane. We note that $H$ is an integer multiple of $\Delta x$. Thus we define an operator at the interface plane as $\hat{w}_{x x}^{n}=\frac{w_{i+I H, j, k}^{n}-2 w_{i, j, k}^{n}+w_{i-I H, j, k}^{n}}{H^{2}}$, where $w_{i+I H, j, k}^{n}$ and $w_{i-I H, j, k}^{n}$ are the approximated values at the adjacent interface planes. Then we propose, in 
this paper, that the values at the interface planes are obtained by the following interface prediction scheme:

$$
\begin{aligned}
w_{t t}^{n}+a_{i j k}^{n} w_{t}^{n}+b_{i j k}^{n} w_{i j k}^{n} & =c_{i j k}^{n} \frac{1}{2}\left[\hat{w}_{x x}^{n+1}+\hat{w}_{x x}^{n-1}\right]+d_{i j k}^{n} \frac{1}{2}\left[w_{y y}^{n+1}+w_{y y}^{n-1}\right] \\
& +e_{i j k}^{n} \frac{1}{2}\left[w_{z z}^{n+1}+w_{z z}^{n-1}\right]+f_{i j k}^{n} .
\end{aligned}
$$

After estimating interface values, each sub-problem is solved by the fully implicit scheme. Then we repeat this process until the last time level. We call the whole procedure the second-order implicit prediction (SIP) method. Stencils of the method at each time level are given in Figure 2. The whole SIP scheme is summarized as the following.

\section{$<$ Second-order Implicit Prediction (SIP) algorithm>}

- Step1: Predict interface values using Equation (6)

$$
\begin{aligned}
& w_{t t}^{n}+a_{i j k}^{n} w_{t}^{n}+b_{i j k}^{n} w_{i j k}^{n}=c_{i j k}^{n} \frac{1}{2}\left[\hat{w}_{x x}^{n+1}+\hat{w}_{x x}^{n-1}\right]+d_{i j k}^{n} \frac{1}{2}\left[w_{y y}^{n+1}+w_{y y}^{n-1}\right] \\
& \quad+e_{i j k}^{n} \frac{1}{2}\left[w_{z z}^{n+1}+w_{z z}^{n-1}\right]+f_{i j k}^{n} \text {, where } \hat{w}_{x x}^{n+1} \text { and } \hat{w}_{x x}^{n-1} \text { are defined earlier }
\end{aligned}
$$

- Step2: Solve interior linear system using FIS (5)

$$
\begin{aligned}
& w_{t t}^{n}+a_{i j k}^{n} w_{t}^{n}+b_{i j k}^{n} w_{i j k}^{n}=c_{i j k}^{n} \frac{1}{2}\left[w_{x x}^{n+1}+w_{x x}^{n-1}\right]+d_{i j k}^{n} \frac{1}{2}\left[w_{y y}^{n+1}+w_{y y}^{n-1}\right] \\
& \quad+e_{i j k}^{n} \frac{1}{2}\left[w_{z z}^{n+1}+w_{z z}^{n-1}\right]+f_{i j k}^{n}
\end{aligned}
$$

- Step3: Repeat Step1 and Step2 until the last time level

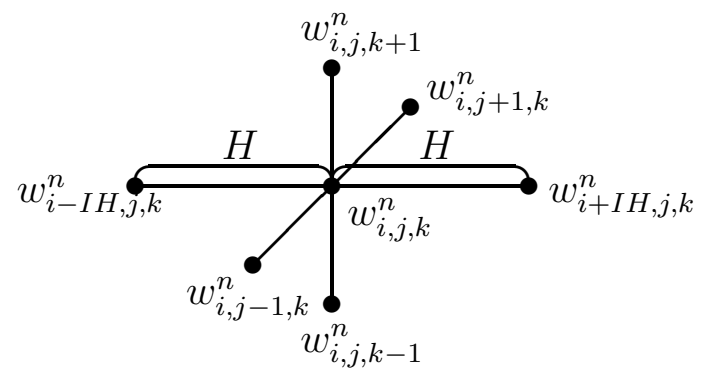

(a) Interface

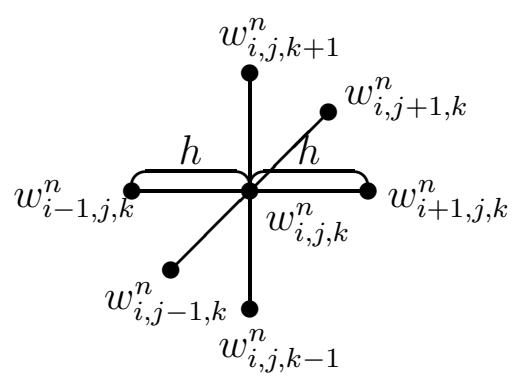

(b) Interior

Figure 2. Stencils of the SIP scheme at each time level

Now we provide a theorem for the unconditional stability and truncation errors of the prediction scheme and the interior scheme of the SIP algorithm, respectively. In the theorem, we see the accuracy of the interface prediction along the $x$-direction of the new method is of second order $H^{2}$.

Theorem 2.1. The interface prediction scheme and the interior scheme of the SIP method are both unconditionally stable and the error terms are $\left|w_{i j k}^{n}-u_{i j k}^{n}\right|=O\left(H^{2}+(\Delta y)^{2}+(\Delta z)^{2}+(\Delta t)^{2}\right)$ and $\left|w_{i j k}^{n}-u_{i j k}^{n}\right|=O\left((\Delta x)^{2}+\right.$ $\left.(\Delta y)^{2}+(\Delta z)^{2}+(\Delta t)^{2}\right)$, respectively. 
Proof. It is well-known [1] that the fully implicit scheme (FIS) is unconditionally stable. The SIP interface scheme is a kind of FIS, in which the step size of the $x$-direction is $H$. Thus, the SIP interface scheme is unconditionally stable. Suppose the domain is decomposed into $P$ subdomains. Then $\hat{w}_{x x}^{n}=u_{x x}+O\left(H^{2}\right), w_{y y}^{n}=u_{y y}+O\left((\Delta y)^{2}\right), w_{z z}^{n}=u_{z z}+O\left((\Delta z)^{2}\right)$, $w_{t t}^{n}=u_{t t}+O\left((\Delta t)^{2}\right)$, and $w_{t}^{n}=u_{t}+O\left((\Delta t)^{2}\right)$. So we can easily see that the truncation error is $\left|w_{i j k}^{n}-u_{i j k}^{n}\right|=O\left(H^{2}+(\Delta y)^{2}+(\Delta z)^{2}+(\Delta t)^{2}\right)$. Note that $H$ is an integer multiple of $\Delta x$. Similarly, the interior scheme of SIP is FIS itself, which explains the unconditional stability. Finally, we clearly see that $\left|w_{i j k}^{n}-u_{i j k}^{n}\right|=O\left((\Delta x)^{2}+(\Delta y)^{2}+(\Delta z)^{2}+(\Delta t)^{2}\right)$.

When we solve the hyperbolic problem using the finite difference method, it is important to approximate the value at the grid point $\left(x_{i}, y_{j}, z_{k}, t_{1}\right)$. We define $\tilde{w}_{x x}=\frac{w_{i+1, j, k}^{0}-2 w_{i j k}^{0}+w_{i-1, j, k}^{0}}{(\Delta x)^{2}}, \tilde{w}_{y y}=\frac{w_{i, j+1, k}^{0}-2 w_{i j k}^{0}+w_{i, j-1, k}^{0}}{(\Delta y)^{2}}, \tilde{w}_{z z}=\frac{w_{i, j, k+1}^{0}-2 w_{i j k}^{0}+w_{i, j, k-1}^{0}}{(\Delta z)^{2}}$. Then we approximate the value $w_{i j k}^{1}$ by

$$
\begin{gathered}
w_{i j k}^{1}=w_{i j k}^{0}+\Delta t(\psi)_{i j k} \\
+\frac{(\Delta t)^{2}}{2}\left[c_{i j k}^{0} \tilde{w}_{x x}+d_{i j k}^{0} \tilde{w}_{y y}+e_{i j k}^{0} \tilde{w}_{z z}-a_{i j j}^{0}(\psi)_{i j k}-b_{i j k}^{0} w_{i j k}^{0}+f_{i j k}^{0}\right] .
\end{gathered}
$$

\section{Numerical Experiments}

In this section, we present the results from our numerical experiments on the proposed second-order implicit prediction (SIP) algorithm. The fully implicit scheme (FIS) is used for our benchmark comparison since it is a well known unconditionally stable scheme. For the numerical experiments, we solve the following two model problems for different values of step sizes. The initial and boundary conditions and $f(x, y, z, t)$ are derived from the exact solution $u=e^{-t} \sinh x \sinh y \sinh z$. All our numerical experiments are carried out on a IBM System x3400 M3 server with Intel Xeon E5630 CPU running at $2.53 \mathrm{GHz}$.

1. Model Problem 1 (MP1):

$$
\begin{aligned}
& u_{t t}+2 e^{x+y+z} u_{t}+\sin ^{2}(x+y+z) u=\left(1+x^{2}\right) u_{x x}+\left(1+y^{2}\right) u_{y y}+(1+ \\
& \left.z^{2}\right) u_{z z}+f(x, y, z, t)
\end{aligned}
$$

2. Model Problem 1 (MP2):

$$
u_{t t}+\frac{2}{x^{2}} u_{t}+\frac{1}{x^{2}} u=\left(1+x^{2}+y^{2}+z^{2}\right)\left(u_{x x}+u_{y y}+u_{z z}\right)+f(x, y, z, t)
$$

First, we investigate the unconditional stability for the SIP scheme. We compare the proposed SIP scheme of 10 subdomains, SIP(10), with the fully explicit scheme (FES) using Equation (4) and the fully implicit scheme (FIS) using Equation (5). Table 1 shows the maximum errors of the model problems 
at the different values of $\lambda$ ranging from $\frac{3}{16}$ to 300 , where $\lambda=\left(\frac{\Delta t}{\Delta x}\right)^{2}+\left(\frac{\Delta t}{\Delta y}\right)^{2}+$ $\left(\frac{\Delta t}{\Delta z}\right)^{2}$. As we can see in Table 1, FES is conditionally stable and FIS and $\operatorname{SIP}(10)$ are unconditionally stable. We should point out that our $\operatorname{SIP}(10)$ scheme is as accurate as FIS or even more accurate than FIS.

Second, we analyze the efficiency of the proposed SIP scheme. One measurement for the efficiency of a parallel algorithm is parallel CPU time (PCPU) which is defined by total CPU time (TCPU) divided by the number of subdomains $(P)$. Table 2 shows maximum error, TCPU, and PCPU of the model problems using the SIP scheme with various $P$ but fixed $\lambda=3$. We see in Table 2 that PCPU decreases significantly when the number of subdomains $P$ increases, which means that $\operatorname{SIP}(P)$ is very efficient.

Table 1. Maximum error of model problems with various $\lambda$

\begin{tabular}{ll|ccc|ccc}
\multicolumn{7}{c}{$\left(\Delta x=\Delta y=\Delta z=\frac{1}{100}\right)$} \\
\hline \multirow{3}{*}{$\Delta t$} & $\lambda$ & \multicolumn{3}{c}{ MP1 } & & MP2 \\
\cline { 3 - 8 } & FES & FIS & SIP $(10)$ & FES & FIS & SIP $(10)$ \\
\hline $1 / 10$ & 300 & $\infty$ & $0.118 \mathrm{e}-3$ & $0.142 \mathrm{e}-3$ & $\infty$ & $0.147 \mathrm{e}-3$ & $0.141 \mathrm{e}-3$ \\
$1 / 20$ & 75 & $\infty$ & $0.101 \mathrm{e}-3$ & $0.512 \mathrm{e}-4$ & $\infty$ & $0.938 \mathrm{e}-4$ & $0.484 \mathrm{e}-4$ \\
$1 / 50$ & 12 & $\infty$ & $0.981 \mathrm{e}-4$ & $0.508 \mathrm{e}-4$ & $\infty$ & $0.846 \mathrm{e}-4$ & $0.483 \mathrm{e}-4$ \\
$1 / 100$ & 3 & $\infty$ & $0.605 \mathrm{e}-4$ & $0.476 \mathrm{e}-4$ & $\infty$ & $0.746 \mathrm{e}-4$ & $0.414 \mathrm{e}-4$ \\
$1 / 400$ & $3 / 16$ & $0.2054 \mathrm{e}-6$ & $0.368 \mathrm{e}-4$ & $0.215 \mathrm{e}-4$ & $0.1373 \mathrm{e}-6$ & $0.572 \mathrm{e}-4$ & $0.164 \mathrm{e}-4$ \\
\hline
\end{tabular}

Table 2. Maximum error and CPU time of SIP with various $P$

\begin{tabular}{cl|cccccc}
\multicolumn{6}{c}{ (TCPU=Total CPU, PCPU=Parallel CPU, $\left.\Delta x=\Delta y=\Delta z=\Delta t=\frac{1}{100}\right)$} \\
\hline \multirow{2}{*}{ MP1 } & Error & $1(=$ FIS $)$ & 5 & 10 & 20 & 25 & 50 \\
& TCPU & 73 & 128 & 179 & 181 & 174 & 114 \\
& PCPU & 73 & 26 & 18 & 9 & 7 & 2 \\
\hline MP2 & Error & $0.746 \mathrm{e}-4$ & $0.447 \mathrm{e}-4$ & $0.414 \mathrm{e}-4$ & $0.511 \mathrm{e}-4$ & $0.436 \mathrm{e}-4$ & $0.519 \mathrm{e}-4$ \\
& TCPU & 123 & 193 & 259 & 238 & 220 & 144 \\
& PCPU & 123 & 39 & 26 & 12 & 9 & 3 \\
\hline
\end{tabular}

\section{Conclusion}

In this paper, we proposed a non-overlapping stripwise domain decomposition scheme for solving three-dimensional linear second-order hyperbolic partial differential equations. The non-overlapping stripwise decomposition generates interface planes in three-dimensional problem. The prediction scheme of the proposed method at the interface plane is an implicit scheme and of second order accuracy. Unconditional stability of the method was analyzed and demonstrated using numerical experiments in this paper. Numerical results show that 
the new method is as accurate as the classical implicit three-level finite difference scheme or even more accurate, but the one is much more efficient than the other. The method performed very well not only at constant coefficient problems, but also at variable coefficient problems and singular problems.

\section{References}

[1] W.F. Ames, Numerical methods for partial differential equations, Academic Press, 1992.

[2] W. Heineken, M. Kunik, The analytical solution of two interesting hyperbolic problems as a test case for a finite volume method with a new grid refinement technique, J. Comput. Appl. Math. 214 (2008), 509-532.

[3] Y. Jun, A stable noniterative Prediction/Correction domain decomposition method for hyperbolic problems, Appl. Math. Comput. 216 (2010), 2286-2292.

[4] Y. Jun, An unconditionally stable implicit high-order domain decomposition method for two-dimensional second-order hyperbolic problem, Appl. Math. Sci. 6 (2012), 3301-3312.

[5] R.M.J. Kramer, C. Pantano, D.I. Pullin, A class of energy stable, highorder finitedifference interface schemes suitable for adaptive mesh refinement of hyperbolic problems, J. Comput. Phys. 226 (2007), 1458-1484.

[6] X. Lai, Y. Yuan, Galerkin alternating-direction method for a kind of three-dimensional nonlinear hyperbolic problems, Comput. Math. Appl. 57 (2009), 384-403.

[7] R.K. Mohanty, M.K. Jain, U. Arora, An unconditionally stable ADI method for the linear hyperbolic equation in three space dimensions, Int. J. Comput. Math. 79 (2002), 133-142.

[8] R.K. Mohanty, An operator splitting technique for an unconditionally stable difference method for a linear three space dimensional hyperbolic equation with variable coefficients, Appl. Math. Comput. 162 (2005), 549557.

[9] M. Yang, Two-time level ADI finite volume method for a class of secondorder hyperbolic problems, Appl. Math. Comput. 215 (2010), 3239-3248.

\section{Received: October, 2012}

